\title{
Current Management of Metastatic Brain Disease
}

\author{
Tulika Ranjan and Lauren E. Abrey \\ Department of Neurology, Memorial Sloan-Kettering Cancer Center, New York, New York 10065
}

\begin{abstract}
Summary: Brain metastases are the most common intracranial tumor in adults. The incidence of metastases is thought to be rising due to better detection and treatment of systemic malignancy. More widespread use and improved quality of MRI may lead to early detection of brain metastases. Available evidence suggests that survival is longer and quality of life improved if brain metastases are treated aggressively. This article reviews current therapeutic management used for brain metastases. To select the appropriate therapy, the physician must consider the extent of the systemic disease, primary histology, and patient age and performance status, as well as the number, size, and location of the brain
\end{abstract}

metastases. Available treatment options include whole-brain radiotherapy (WBRT), stereotactic radiosurgery (SRS), surgery, and chemotherapy. Multidisciplinary approaches such as the combination of WBRT with SRS or surgery have shown superior results in terms of survival time, neurocognitive function, and quality of life. The utility and optimal use of chemotherapy and radiosensitizing agents is less clear. It is hoped that further advances and multidisciplinary approaches currently under study will result in improved patient outcomes. Key Words: Brain metastases, brain metastasis chemotherapy, brain metastasis radiotherapy, metastatic brain tumor, brain neoplasm.

\section{INTRODUCTION}

Brain metastases are the most common intracranial tumor in adults; the current incidence is estimated to be more than 200,000 cases annually in the United States. In patients with systemic malignancies, brain metastases occur in $10 \%$ to $30 \%$ of adults and in $6 \%$ to $10 \%$ of children. Autopsy studies reveal intracranial metastasis in approximately $25 \%$ of patients who die of cancer. ${ }^{1-6}$ Most brain metastases originate from one of three primary malignancies: lung cancer (40\% to $50 \%)$, breast cancer ( $15 \%$ to $25 \%$ ), and melanoma (5\% to $20 \%$ ).

Lung cancer, which is the primary tumor most often associated with brain metastasis, accounts for approximately one half of all patients diagnosed with brain metastases in the United States each year. Although nonsmall cell lung cancer accounts for most of this estimate, patients with small cell lung cancer are at particularly high risk of CNS metastasis, approaching $80 \%{ }^{7}$

Breast cancer, the second most common tumor associated with brain metastases, is notable for the risk of late CNS metastases appearing years after the initial diagnosis. Risk factors for brain metastases in the breast cancer

Address correspondence and reprint requests to: Lauren E. Abrey, M.D., Department of Neurology, Memorial Sloan-Kettering Cancer Center, 1275 York Avenue, New York, NY 10065. E-mail: abreyl @ mskcc.org. population include young age, negative estrogen receptors, premenopausal status, infiltrating ductal carcinoma histology, BRCAl mutation, altered p53 and positive lymph nodes. The use of trastuzumab appears to be associated with an increased risk of brain metastases as the first site of progressive disease; estimates of increased risk are complicated by the fact that HER-2 positivity is itself a risk factor for developing brain metastases. ${ }^{8}$ However, approximately one third of women receiving trastuzumab for metastatic breast cancer develop CNS metastases. $^{8-10}$

Brain metastases from melanoma are the third most common cause of brain metastases. Less commonly, other primary tumors are responsible, such as colorectal cancers, germ cell tumors, non-Hodgkin lymphoma, and ovarian and kidney cancers; however, carcinomas of the prostate, esophagus, and oropharynx and nonmelanoma skin cancers rarely metastasize to the brain.

The incidence of brain metastasis is thought to be increasing, although supporting evidence is limited. It is likely that improved therapies for systemic malignancy have resulted in prolonged survival, thereby increasing the risk for brain metastases in select patients. Furthermore, brain metastases present more often in the setting of well-controlled systemic disease and are therefore more likely to be treated than in the historic paradigm, wherein brain metastases develop in concert with multiorgan metastatic disease near the end of life. Finally, 


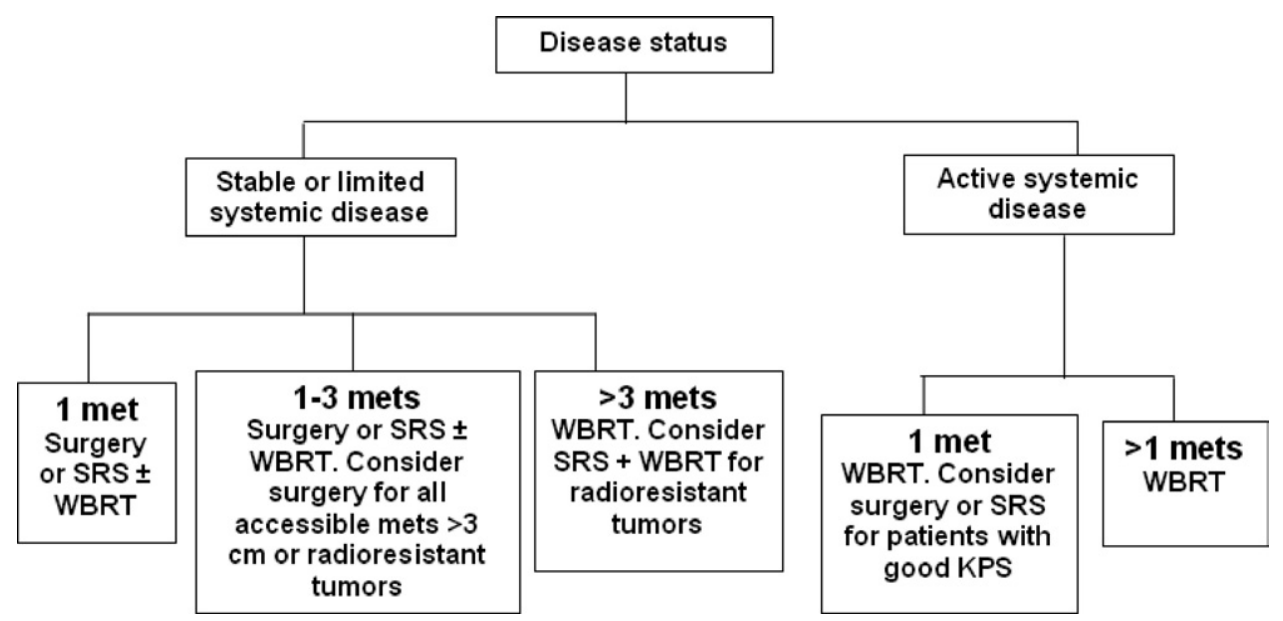

FIG. 1. Approach to the treatment of brain metastases. KPS $=$ Karnofsky performance status; met $=$ metastasis; SRS $=$ stereotactic radiosurgery; WBRT $=$ whole-brain radiation therapy.

improved imaging and increased access to such imaging further increase the likelihood of discovering and diagnosing occult brain metastases, particularly in view of several new National Comprehensive Cancer Network $(\mathrm{NCCN})$ guidelines recommending screening MRI prior to administration of agents such as bevacizumab.

Unfortunately, the diagnosis of brain metastasis continues to portend a poor prognosis for the vast majority of patients. Although some young patients with single metastases and controlled systemic disease may achieve a good outcome, most patients have an expected survival measured in months. Available therapies include surgery, radiotherapy, and chemotherapy; use of radiosensitizers and novel therapies are under active study.

\section{MANAGEMENT}

The management of brain metastases can be subdivided into symptomatic or therapeutic strategies. Symptomatic treatment includes steroids to decrease the vasogenic edema surrounding the tumor. Use of corticosteroids can result in rapid symptomatic and functional improvement, allowing a patient to tolerate aggressive antitumor therapy. When administering corticosteroids, caution must be exercised to minimize associated toxicity, particularly steroid-induced diabetes mellitus and Pneumocystis carinii pneumonia. Anticonvulsants should be used only in patients who have had a seizure. No evidence supports seizure prophylaxis in patients with brain tumors and no history of seizures, regardless of neoplastic type. ${ }^{11}$ Furthermore, anticonvulsants metabolized by the CYP3A4 pathway (including phenytoin, carbamazepine, and phenobarbital) can have significant drug interactions with various chemotherapeutic agents, negating the efficacy of active chemotherapy and should be avoided if possible.
Therapeutic management of patients with brain metastases includes whole-brain radiotherapy (WBRT), stereotactic radiosurgery (SRS), surgery, and chemotherapy (FIG. 1). To select the appropriate therapy, the physician must consider the extent of the systemic disease and the brain metastasis, including the number of brain metastases, their size, location, and histology, and the patient's age and performance status. Available evidence suggests that survival is longer and quality of life is better if brain metastases are treated aggressively.

\section{RADIATION THERAPY}

\section{Whole-brain radiation therapy}

Whole-brain radiation therapy (WBRT), historically the standard of care for brain metastases, remains the most frequently used treatment modality. In particular, WBRT is indicated in patients with multiple metastases $(>3)$, in patients with one to three metastases that are too large for radiosurgery, or for patients who had prior surgery or radiation with progression of disease. Use of WBRT after surgery or radiosurgery of a single brain metastasis has been definitively shown to improve local tumor control. ${ }^{12,13}$ Metastases from the breast or lung are more likely to respond clinically and radiographically to WBRT. ${ }^{14}$ Radioresistant tumors such as melanoma ${ }^{15}$ and colon $^{16}$ and renal ${ }^{17}$ carcinoma are less likely to have a good response to WBRT.

Nonrandomized studies suggest that WBRT increases the median survival time by 3 to 4 months. ${ }^{18}$ Patient survival is approximately 1 month without any treatment and 2 months with corticosteroids alone. The median survival after WBRT varies from 2.3 months to 13.5 months, depending on prognostic factors. ${ }^{19} \mathrm{~A}$ threetiered prognostic scoring system is frequently used in patients treated with WBRT. This was derived from the 
Table 1. Median Survival Duration According to RPA Class for Patients Treated with WBRT

\begin{tabular}{llr}
\hline RPA Class & \multicolumn{1}{c}{ Clinical Characteristics } & Median Survival, mo \\
\hline 1 & KPS score $\geq 70$ AND age $<65$ yr AND controlled primary tumor & 7.1 \\
2 & AND no extra cranial metastases & 4.2 \\
& KPS score $\geq 70$ AND age $\geq 65$ oR uncontrolled primary tumor & 2.3 \\
3 & OR extracranial metastases & \\
\hline
\end{tabular}

Adapted from Gaspar et al. ${ }^{20}$ (Int J Radiat Oncol Biol Phys 1997;37:745-751), with permission.

$\mathrm{KPS}=$ Karnofsky performance status; RPA $=$ recursive partitioning analysis; WBRT $=$ whole-brain radiation therapy.

Radiation Therapy Oncology Group (RTOG) database using recursive partitioning analysis (RPA) and has been validated in multiple consecutive studies (Table $1^{20}$ ).

Of the several fractionation schedules used in the treatment of brain metastases, 30 Gy delivered in 10 fractions is the most common. ${ }^{21}$ Dose escalation beyond 30 Gy does not increase the survival or local control in patients with multiple brain metastases. However, daily fractions of more than 3 Gy may increase the risk for neurotoxicity. ${ }^{19}$ Initiation of corticosteroids 48 to 72 hours before the first dose of WBRT will minimize treatment-emergent side effects such as headache, nausea, and neurologic deterioration. A range of steroid doses can be used, depending on edema, location of the tumor, and clinical symptoms; steroid treatment should be tapered as tolerated after the radiation.

Numerous agents have been tested as potential radiosensitizers to try to improve the efficacy of WBRT, with limited success. The following radiosensitizers have been studied in randomized controlled trials: motexafin gadolinium, ${ }^{22}$ RSR13 (efaproxiral) ${ }^{23}$ celecoxib,${ }^{24}$ paclitaxel, ${ }^{25}$ lonidamine, ${ }^{26}$ metronidazole,${ }^{27}$ misonidazole, ${ }^{28}$ and bromodeoxyuridine. ${ }^{29}$ In a phase III trial, motexafin gadolinium (MGd) improved time to neurologic progression in all patients (median, 4.3 months for MGd vs 3.8 months for WBRT; $p=0.018$ ); in lung cancer patients, the time to neurologic progression was longer (median, 5.5 months for MGd vs 3.7 months for WBRT; $p=$ 0.025). The overall results did not, however, demonstrate significant differences by treatment arm for survival. ${ }^{22}$ Results from another phase III trial suggested that addition of the noncytotoxic radiation sensitizer efaproxiral to WBRT improved response rates and survival in patients with breast cancer and brain metastases. ${ }^{23}$ Additional studies are ongoing, and currently no radiosensitizing agent is approved for the treatment of brain metastases.

The cognitive effects of WBRT are often cited as a concern and as a potential reason to avoid or defer WBRT, particularly in patients with a single brain metastasis treated with surgical resection or stereotactic radiosurgery (SRS). The available data are limited. There is clear evidence that administration of WBRT immediately after SRS or surgical resection improves tumor control in the brain nearly three-fold. ${ }^{13}$ Furthermore, studies of cognitive function after WBRT suggest that neurocognitive outcome is most strongly associated with tumor control in the brain. ${ }^{18}$ Nonetheless, several studies reporting significant neurocognitive decline in long-term survivors after WBRT for brain metastases continue to influence some treatment decisions. ${ }^{30,31}$

\section{Stereotactic radiosurgery}

Stereotactic radiosurgery (SRS) delivers a relatively large single dose of three-dimensional radiation to a small intracranial target with great accuracy. The three most common delivery systems are the linear particle accelerator, the gamma knife, and the cyclotron, which make use of high-energy photons, gamma rays, and protons, respectively. Normally, SRS is reserved for patients with a single or two to three brain metastases, metastases less than $3 \mathrm{~cm}$ in maximum diameter, highly radioresistant tumors, and metastases inaccessible for surgical resection.

In selected patients with radioresistant tumors, such as melanoma, sarcoma and renal cell carcinoma, more than three metastatic lesions may be treated with SRS. Significant prognostic factors for survival in patients receiving SRS are Karnofsky performance status (KPS) of 70 or higher, no extracerebral disease, pretreatment neurological functional class, histology (renal cell and breast carcinomas), and dose to the planning target volume (20 Gy and higher). ${ }^{32}$ One study demonstrated a local control rate of $85 \%$ for tumors less than $2 \mathrm{~cm}$; this fell to $45 \%$ for tumors greater than $3 \mathrm{~cm} .{ }^{33}$

Large metastases and metastases associated with extensive edema can be difficult to control with radiosurgery because of a high risk of radiation necrosis or neurologic deterioration at biologically effective doses. ${ }^{34}$ Two courses of SRS are rarely administered to the same site because of the risk of radionecrosis. In a retrospective analysis of 111 patients harboring 238 metastatic brain lesions, 19 of the 120 lesions treated with SRS alone (16\%) and 26 of the 114 treated with WBRT plus SRS $(23 \%)$ developed radiation necrosis. Of the lesions that received $20 \mathrm{~Gy}, 18 \mathrm{~Gy}$, and $15 \mathrm{~Gy}$ treatment, 20\%, $20 \%$, and $12 \%$ developed radiation necrosis, respectively. ${ }^{35}$ 
Table 2. Prospective Studies Comparing Surgery or SRS Plus WBRT versus WBRT Alone

\begin{tabular}{|c|c|c|c|}
\hline Study and Treatment & $\mathrm{N}$ & $\begin{array}{c}\text { Median } \\
\text { Survival, mo }\end{array}$ & $p$-Value \\
\hline \multicolumn{4}{|l|}{ Patchell et al., ${ }^{38} 1990$} \\
\hline Surgery + WBRT & 25 & 9.2 & \multirow[t]{2}{*}{$<0.01$} \\
\hline WBRT & 23 & 3.4 & \\
\hline \multicolumn{4}{|l|}{ Vecht et al., ${ }^{39} 1993$} \\
\hline Surgery + WBRT & 32 & 10 & \multirow[t]{2}{*}{0.04} \\
\hline WBRT & 31 & 6 & \\
\hline \multicolumn{4}{|l|}{ Mintz et al., ${ }^{40} 1996$} \\
\hline Surgery + WBRT & 41 & 5.6 & \multirow[t]{2}{*}{0.24} \\
\hline WBRT & 43 & 6.3 & \\
\hline \multicolumn{4}{|l|}{ Andrews et al., ${ }^{41} 2004$} \\
\hline $\mathrm{SRS}+\mathrm{WBRT}$ & 167 & 6.5 & \multirow[t]{2}{*}{0.13} \\
\hline WBRT & 164 & 5.7 & \\
\hline \multicolumn{4}{|c|}{ Kondziolka et al., ${ }^{42} 1999$} \\
\hline SRS + WBRT & 13 & 11 & \multirow[t]{2}{*}{0.22} \\
\hline WBRT & 14 & 7.5 & \\
\hline
\end{tabular}

$\mathrm{SRS}=$ stereotactic radiosurgery; WBRT $=$ whole-brain radiation therapy.

Dosing in SRS is determined by the volume of tumor irradiated, and the maximum tolerated doses of 15,18 , and 24 Gy have been established for the tumor sizes $31-40 \mathrm{~mm}, 21-30 \mathrm{~mm}$, and $\leq 20 \mathrm{~mm}$ maximum diameter, respectively, according to the RTOG protocol 9005. Vogelbaum et al. $^{33}$ found that brain metastases treated with SRS to a dose of $24 \mathrm{~Gy}$ to the tumor margin had a significantly lower risk of local failure than 15 or $18 \mathrm{~Gy}(p=0.0005$; hazard ratio $\mathrm{HR}=0.277$, confidence interval $\mathrm{CI}=0.134-0.573$ ), whereas the $15-\mathrm{Gy}$ and 18-Gy groups were not significantly different from each other $(p=0.82)$. The 1-year local control rate was $85 \%$ $(95 \% \mathrm{CI}=78-92)$ in tumors treated with $24 \mathrm{~Gy}$, compared with $49 \%(95 \% \mathrm{CI}=30-68)$ in tumors treated with $18 \mathrm{~Gy}$ and $45 \%(95 \% \mathrm{CI}=23-67)$ in tumors treated with $15 \mathrm{~Gy}^{33}$

The addition of WBRT to SRS may result in improved local and CNS control rates (Table 2). Although there is no evidence of a survival advantage for patients with one to four metastases undergoing SRS or SRS plus WBRT, there is evidence that, in RPA class 1 and class 2 patients with one to three metastases, SRS plus WBRT is associated with improved local tumor control and improved KPS score, compared with WBRT alone. Furthermore, there is evidence that in patients with a single metastasis treated with SRS, the addition of WBRT results in improved survival. In RPA class 1 and class 2 patients with one to four metastases, SRS plus WBRT is associated with improved tumor control. ${ }^{36}$

\section{SURGERY}

Surgery can serve multiple purposes in a patient with brain metastases. Obtaining tissue for diagnostic pur- poses is critical in a patient who presents with an apparent brain metastasis and no known systemic malignancy or accessible systemic tumor for biopsy. Patients with a known primary malignancy who have been in a sustained remission or have a histology with a low likelihood for CNS metastases are at risk of a new primary tumor and should also be referred to a neurosurgeon to obtain histologic confirmation prior to initiation of definitive therapy.

Surgery can also serve as a valuable therapeutic modality in the management of brain metastases. In particular, patients with large single lesions may derive immediate neurologic improvement in function, and adjuvant therapy is more likely to be effective in patients with minimal tumor burden. Prolonged survival is observed in a proportion of patients after surgical resection, with one series reporting a median survival of 2 years and $22 \%$ of patients alive at 4 years. ${ }^{37}$ Several prospective studies have clearly demonstrated that patients with a single metastasis and good prognostic features benefit from surgery followed by WBRT (Table 2). ${ }^{38-42}$

Surgery can also be used as a delivery method for other therapies. Implantation of radioactive seeds (i.e., brachytherapy) has been used for treating brain metastases both initially and at recurrence. A high rate of local tumor control can be achieved by brachytherapy, with a low risk of radiation-induced morbidity. Currently, the use of brachytherapy is largely reserved for tumors that have recurred despite prior focal irradiation and those that are too large (between 2 and $5 \mathrm{~cm}$ in greatest diameter) for safe treatment with SRS. Excellent local control has been achieved using permanent iodine- 125 brachytherapy for brain metastasis resection cavities-although there is a high risk of radiation necrosis over time. ${ }^{43}$ The addition of local chemotherapy delivered via carmustine polymer wafers to a regimen of surgical resection and external beam radiotherapy was well tolerated by patients undergoing surgery for a single brain metastasis. ${ }^{44}$

\section{CHEMOTHERAPY}

The role of chemotherapy in the management of brain metastases is fairly limited at present. Brain metastases are relatively chemoresistant and often develop after use of the most effective chemotherapeutic agents in a given patient. Furthermore, delivery of adequate doses can be challenging because of the blood-brain barrier and because of interactions with concomitant medications. Finally, the tumor clone that metastasizes to the CNS may have molecular and genotypic alterations that render brain metastases more resistant. Despite these limitations, a number of drugs have been studied or reported with some success, most often in patients with recurrent brain metastases after standard therapy (Table $3^{45-57}$ ). The most appropriate use of chemotherapy is to try to 
Table 3. Chemotherapy Regimens Reported for Brain Metastases

\begin{tabular}{|c|c|c|c|}
\hline Cancer and Treatment & $\mathrm{N}$ & Objective Response Rate, \% & Median Overall Survival \\
\hline \multicolumn{4}{|l|}{ Non-small cell lung cancer } \\
\hline temozolomide + cisplatin + WBRT $^{45}$ & 50 & 16 & $5 \mathrm{mo}$ \\
\hline gefitinib $^{46}$ & 40 & 32 & $15 \mathrm{mo}$ \\
\hline gefitinib $^{47}$ & 76 & 33.3 & $9.9 \mathrm{mo}$ \\
\hline vinorelbine, gemcitabine, carboplatin ${ }^{48}$ & 22 & 45 & $33 \mathrm{wk}$ \\
\hline cisplatin, ifosfamide, irinotecan ${ }^{49}$ & 30 & 50 & $382 \mathrm{~d}$ \\
\hline \multicolumn{4}{|l|}{ Breast cancer } \\
\hline $\mathrm{CFP}^{50}$ & 52 & 52 & * \\
\hline CFP-MV 50 & 35 & 54 & * \\
\hline $\mathrm{MVP}^{50}$ & 7 & 43 & $*$ \\
\hline adriamycin-cyclophosphamide $^{50}$ & 6 & 17 & * \\
\hline $\mathrm{CMF}(\text { or } \mathrm{CAF})^{51}$ & $20(2)$ & 59 & 25 wk \\
\hline cisplatin + etoposide $^{52}$ & 56 & 38 & $31 \mathrm{wk}$ \\
\hline temozolomide $^{53}$ & 10 & 40 (SD) & not reported \\
\hline high-dose methotrexate ${ }^{54}$ & 29 & 28 & $20 \mathrm{wk}$ \\
\hline \multicolumn{4}{|l|}{ Melanoma } \\
\hline cisplatin, vinblastine, temozolomide, INF- $\alpha$, IL- $2^{55}$ & 5 & 20 & $1 \mathrm{CR} ; 2 \mathrm{SD}$ \\
\hline temozolomide + thalidomide + WBRT $^{56}$ & 39 & 76 & $4 \mathrm{mo}$ \\
\hline temozolomide $^{57}$ & 6 & 24 & $4.7 \mathrm{mo}$ \\
\hline temozolomide + docetaxel ${ }^{57}$ & 10 & 24 & $4.7 \mathrm{mo}$ \\
\hline temozolomide + cisplatin $^{57}$ & 9 & 24 & $4.7 \mathrm{mo}$ \\
\hline
\end{tabular}

$\mathrm{CAF}=$ cyclophosphamide-adriamycin-5-fluorouracil; $\mathrm{CFP}=$ cyclophosphamide-5-fluorouracil-prednisone; $\mathrm{CMF}=$ cyclophosphamidemethotrexate-5-fluorouracil; $\mathrm{CR}=$ complete response; IL = interleukin; INF = interferon; $\mathrm{MV}=$ methotrexate and vincristine; $\mathrm{MVP}=$ methotrexate-vincristine-platinum compound; $\mathrm{SD}=$ stable disease; WBRT $=$ whole-brain radiation therapy.

*Median overall survival was 39.5 mo for patients with complete response and 10.5 mo for patients with partial response.

salvage a patient with recurrent or progressive brain metastases after standard therapy. In select patients with asymptomatic brain metastases at diagnosis, it may be reasonable to administer systemic chemotherapy prior to definitive brain therapy, to control symptomatic systemic disease. No specific recommendation can be made with regard to selecting a particular chemotherapeutic agent; consideration should be given to the most effective agent for a particular histology and to the ability of this agent to penetrate the blood-brain barrier. In addition, there is an increasing interest in developing clinical trials to test a variety of novel agents for patients with brain metastases.

\section{CONCLUSIONS}

Although the management of brain metastases remains challenging and often discouraging, a careful approach to optimizing management may result in substantial benefit to the patient, with prolonged survival or freedom from neurologic deterioration in select patients. A multidisciplinary assessment including medical and neuro-oncology, radiation oncology, neurosurgery, and neurology is advised for developing an integrated approach. Referral to appropriate clinical trials may enhance care and advance treatment options for this difficult disease.

\section{REFERENCES}

1. Patchell R. Brain metastases. Handb Neurol 1997;25:135.
2. Posner JB. Management of brain metastases. Rev Neurol (Paris) 1992;148:477-487.

3. Sawaya R, Bindal RK. Metastatic brain tumors. 2nd ed. In: Kaye AH, Laws ER, editors. Brain tumors. Edinburgh: Churchill Livingstone; 2001:999-1026.

4. Graus F, Walker RW, Allen JC. Brain metastases in children. J Pediatr 1983;103:558-561.

5. Wen PY, Loeffler JS. Management of brain metastases. Oncology 1999;13:941-954.

6. Johnson JD, Young B. Demographics of brain metastasis. Neurosurg Clin N Am 1996;7:337-344.

7. Raizer JJ, Abrey LE, editors. Brain metastases. New York: Springer, 2007.

8. Lin NU, Winer EP. Brain metastases: the HER2 paradigm. Clin Cancer Res 2007;13:1648-1655.

9. Stemmler J, Schmitt M, Willems A, et al. Brain metastases in HER-2 overexpressing metastatic breast cancer: comparative analysis of trastuzumab levels in serum and cerebrospinal fluid. J Clin Oncol 2006;24:64s (abstract).

10. Pestalozzi BC, Brignoli S. Trastuzumab in CSF. J Clin Oncol 2000;18:2349-2351.

11. Sirven JI, Wingerchuk DM, Drazkowski JF, Lyons MK, Zimmerman RS. Seizure prophylaxis in patients with brain tumors: a meta-analysis. Mayo Clin Proc 2004;79:1489-1494.

12. Shehata MK, Young B, Reid B, et al. Stereotactic radiosurgery of 468 brain metastases $\leq 2 \mathrm{~cm}$ : implications for SRS dose and whole brain radiation therapy. Int J Radiat Oncol Biol Phys 2004;59: 87-93.

13. Patchell RA, Tibbs PA, Regine WF, et al. Postoperative radiotherapy in the treatment of single metastases to the brain: a randomized trial. JAMA 1998;280:1485-1489.

14. Cairncross JG, Kim JH, Posner JB. Radiation therapy for brain metastases. Ann Neurol 1980;7:529-541.

15. Byrne TN, Cascino TL, Posner JB. Brain metastasis from melanoma. J Neurooncol 1983;1:313-317.

16. Cascino TL, Leavengood JM, Kemeny N, Posner JB. Brain metastases from colon cancer. J Neurooncol 1983;1:203-209.

17. Gay PC, Litchy WJ, Cascino TL. Brain metastasis in hypernephroma. J Neurooncol 1987;5:51-56. 
18. Li J, Bentzen SM, Renschler M, Mehta MP. Regression after whole-brain radiation therapy for brain metastases correlates with survival and improved neurocognitive function. J Clin Oncol 2007; 25:1260-1266.

19. Rades D, Haatanen T, Schild SE. Dose escalation beyond 30 grays in 10 fractions for patients with multiple brain metastases. Cancer 2007;110:1345-1350.

20. Gaspar L, Scott C, Rotman M, et al. Recursive partitioning analysis (RPA) of prognostic factors in three Radiation Therapy Oncology Group (RTOG) brain metastases trials. Int J Radiat Oncol Biol Phys 1997;37:745-751.

21. Kurtz JM, Gelber R, Brady LW, Carella RJ, Cooper JS; Radiation Therapy Oncology Group. The palliation of brain metastases in a favorable patient population: a randomized clinical trial by the Radiation Therapy Oncology Group. Int J Radiat Oncol Biol Phys 1981;7:891-895.

22. Mehta MP, Rodrigus P, Terhaard CH, et al. Survival and neurologic outcomes in a randomized trial of motexafin gadolinium and whole-brain radiation therapy in brain metastases. J Clin Oncol 2003;21:2529-2536.

23. Suh JH, Stea B, Nabid A, et al. Phase III study of efaproxiral as an adjunct to whole-brain radiation therapy for brain metastases. J Clin Oncol 2006;24:106-114.

24. Cerchietti LC, Bonomi MR, Navigante AH, Castro MA, Cabalar ME, Roth BM. Phase I/II study of selective cyclooxygenase-2 inhibitor celecoxib as a radiation sensitizer in patients with unresectable brain metastases. J Neurooncol 2005;71:73-81.

25. Tishler RB, Schiff PB, Geard CR, Hall EJ. Taxol: a novel radiation sensitizer. Cancer Res 1992;52:3495-3497.

26. DeAngelis LM, Currie VE, Kim JH, et al. The combined use of radiation therapy and lonidamine in the treatment of brain metastases. J Neurooncol 1989;7:241-247.

27. Eyre HJ, Ohlsen JD, Frank J, et al; Southwest Oncology Group Randomized trial of radiotherapy versus radiotherapy plus metronidazole for the treatment metastatic cancer to brain. A Southwest Oncology Group study. J Neurooncol 1984;2:325-330.

28. Komarnicky LT, Phillips TL, Martz K, Asbell S, Isaacson S, Urtasun R. A randomized phase III protocol for the evaluation of misonidazole combined with radiation in the treatment of patients with brain metastases (RTOG-7916). Int J Radiat Oncol Biol Phys 1991;20:53-58.

29. Phillips TL, Scott CB, Leibel SA, Rotman M, Weigensberg IJ. Results of a randomized comparison of radiotherapy and bromodeoxyuridine with radiotherapy alone for brain metastases: report of RTOG trial 89-05. Int J Radiat Oncol Biol Phys 1995;33:339-348.

30. Conill C, Berenguer J, Vargas M, et al. Incidence of radiationinduced leukoencephalopathy after whole brain radiotherapy in patients with brain metastases. Clin Transl Oncol 2007;9:590-595.

31. DeAngelis LM, Delattre JY, Posner JB. Radiation-induced dementia in patients cured of brain metastases. Neurology 1989;39:789796.

32. Simonová G, Liscák R, Novotný J Jr, Novotný J. Solitary brain metastases treated with the Leksell gamma knife: prognostic factors for patients. Radiother Oncol 2000;57:207-213.

33. Vogelbaum MA, Angelov L, Lee SY, Li L, Barnett GH, Suh JH. Local control of brain metastases by stereotactic radiosurgery in relation to dose to the tumor margin. J Neurosurg 2006;104:907912.

34. Varlotto JM, Flickinger JC, Niranjan A, Bhatnagar AK, Kondziolka D, Lunsford LD. Analysis of tumor control and toxicity in patients who have survived at least one year after radiosurgery for brain metastases. Int J Radiat Oncol Biol Phys 2003;57:452-464.

35. Xu N, Shu H, Marcus R, Crocker IR. Radiation necrosis after stereotactic radiosurgery for metastatic brain disease. Int $\mathrm{J}$ Radiat Oncol Biol Phys 2007;69:S163 (abstract).

36. Müller-Riemenschneider F, Bockelbrink A, et al. Stereotactic radiosurgery for the treatment of brain metastases. Radiother Oncol 2009;91:67-74.

37. Moser RP, Johnson ML, Surgical management of brain metastases: how aggressive should we be? Oncology 1989;3:123-127.
38. Patchell RA, Tibbs PA, Walsh JW, et al. A randomized trial of surgery in the treatment of single metastases to the brain. N Engl J Med 1990;322:494-500.

39. Vecht CJ, Haaxma-Reiche H, Noordijk EM, et al. Treatment of single brain metastasis: radiotherapy alone or combined with neurosurgery? Ann Neurol 1993;33:583-590.

40. Mintz AH, Kestle J, Rathbone MP, et al. A randomized trial to assess the efficacy of surgery in addition to radiotherapy in patients with a single cerebral metastasis. Cancer 1996;78:1470-1476.

41. Andrews DW, Scott CB, Sperduto PW, et al. Whole brain radiation therapy with or without stereotactic radiosurgery boost for patients with one to three brain metastases: phase III results of the RTOG 9508 randomised trial. Lancet 2004;363:1665-1672.

42. Kondziolka D, Patel A, Lunsford LD, et al. Stereotactic radiosurgery plus whole brain radiotherapy versus radiotherapy alone for patients with multiple brain metastases. Int J Radiat Oncol Biol Phys 1999;45:427-434.

43. Huang K, Sneed PK, Kunwar S, et al. Surgical resection and permanent iodine- 125 brachytherapy for brain metastases. J Neurooncol 2009;91:83-93.

44. Ewend MG, Brem S, Gilbert M, et al. Treatment of single brain metastasis with resection, intracavity carmustine polymer wafers, and radiation therapy is safe and provides excellent local control. Clin Cancer Res 2007;13:3637-3641.

45. Cortot AB, Gerinière L, Robinet G, et al. Groupe Lyon-SaintEtienne d'Oncologie Thoracique; Groupe Français de PneumoCancérologie. Phase II trial of temozolomide and cisplatin followed by whole brain radiotherapy in non-small-cell lung cancer patients with brain metastases: a GLOT-GFPC study. Ann Oncol 2006; 17:1412-1417.

46. Wu C, Li YL, Wang ZM, Li Z, Zhang TX, Wei Z. Gefitinib as palliative therapy for lung adenocarcinoma metastatic to the brain. Lung Cancer 2007;57:359-364.

47. Chiu CH, Tsai CM, Chen YM, Chiang SC, Liou JL, Perng RP. Gefitinib is active in patients with brain metastases from non-small cell lung cancer and response is related to skin toxicity. Lung Cancer 2005;47:129-138.

48. Bernardo G, Cuzzoni Q, Strada MR, et al. First-line chemotherapy with vinorelbine, gemcitabine, and carboplatin in the treatment of brain metastases from non-small-cell lung cancer: a phase II study. Cancer Invest 2002;20:293-302.

49. Fujita A, Fukuoka S, Takabatake H, Tagaki S, Sekine K. Combination chemotherapy of cisplatin, ifosfamide, and irinotecan with rhG-CSF support in patients with brain metastases from non-small cell lung cancer. Oncology 2000;59:291-295.

50. Rosner D, Nemoto T, Lane WW. Chemotherapy induces regression of brain metastases in breast carcinoma. Cancer 1986;58:832839 .

51. Boogerd W, Dalesio O, Bais EM, van der Sande JJ. Response of brain metastases from breast cancer to systemic chemotherapy. Cancer 1992;69:972-978.

52. Franciosi V, Cocconi G, Michiara M, et al. Front-line chemotherapy with cisplatin and etoposide for patients with brain metastases from breast carcinoma, non-small cell lung carcinoma, or malignant melanoma: a prospective study. Cancer 1999;85:1599-1605.

53. Abrey LE, Olson JD, Raizer JJ, et al. A phase II trial of temozolomide for patients with recurrent or progressive brain metastases. J Neurooncol 2001;53:259-265.

54. Lassman AB, Abrey LE, Shah GD, et al. Systemic high-dose intravenous methotrexate for central nervous system metastases. J Neurooncol 2006;78:255-260.

55. Ron IG, Sarid D, Ryvo L, et al. A biochemotherapy regimen with concurrent administration of cisplatin, vinblastine, temozolomide (Temodal), interferon-alfa and interleukin-2 for metastatic melanoma: a phase II study. Melanoma Res 2006;16:65-69.

56. Atkins MB, Sosman JA, Agarwala S, et al. Temozolomide, thalidomide, and whole brain radiation therapy for patients with brain metastasis from metastatic melanoma: a phase II Cytokine Working Group study. Cancer 2008:113:2139-2145.

57. Bafaloukos D, Tsoutsos D, Fountzilas G, et al. The effect of temozolomide-based chemotherapy in patients with cerebral metastases from melanoma. Melanoma Res 2004;14:289-294. 